\title{
Targeting the SPHK1/HIF1 Pathway to Inhibit Colorectal Cancer Stem Cells Niche
}

\author{
Saeideh Gholamzadeh Khoei ${ }^{1,2} \cdot$ Hamid Sadeghi $^{3} \cdot$ Fateme Karimi Dermani $^{1}$
}

Published online: 16 May 2019

(C) Springer Science+Business Media, LLC, part of Springer Nature 2019

Colorectal cancer (CRC) is one of the most common malignancies worldwide and is one of the major leading causes of death [1]. Many therapeutic methods have been used against colorectal cancer, e.g., chemotherapy (such as 5FU, oxaliplatin, leucovorin), and radiotherapy $[2,3]$, but despite therapeutic advances, drug resistance and metastases still occur in a high proportion of patients and the treatment process fails [4]. Several factors cause resistance in cancer cells, such as drug inactivation, drug target alteration, drug efflux, DNA damage repair, cell death inhibition, and the epithelialmesenchymal transition or any combination of these mechanisms [5]. Recently, a small sub-population of tumor cells, termed cancer stem cells (CSCs), with infinite self-renewal potential and the capacity to differentiate into the diverse populations of cells that comprise a tumor, has caught the attention of researchers. They represent the root of cancer that must be eradicated in order to cure it [6]. Increasing evidence indicates that CSCs contribute to drug resistance because the intrinsic characteristics of CSCs include DNA repair capability and regulation of the survival pathway and its extrinsic characteristics or niche microenvironment include hypoxic conditions $[7,8]$. Furthermore, many of the normal stem cell pathways such as the Hedgehog (Hh), Notch, and Wnt signaling pathways, which guide cellular proliferation, differentiation, and apoptosis, are also prominent in CSCs [9] while the hypoxia pathway is specific to CSCs and niche. Hypoxia occurs in growing tumors or when access to $\mathrm{O}_{2}$ in blood vessels is limited, and the hypoxia pathway guarantees the survival of

Fateme Karimi Dermani

fatemekarimi2007@yahoo.com

1 Research Center for Molecular Medicine, Hamadan University of Medical Sciences, Hamadan, Iran

2 Department of Medical Biotechnology, School of Advanced Medical Sciences and Technologies, Hamadan University of Medical Sciences, Hamadan, Iran

3 Mehr Clinical Diagnostic Laboratory, Qazvin, Iran the tumor. The hypoxia pathway is involved in angiogenesis, metastasis, invasion, and drug resistance [10]. Hypoxiainducible factor (HIF-1 $\alpha$ ), a key transcriptional factor that plays a critical role in hypoxia-related signaling pathway, increases the expression of cell survival or anti-apoptotic genes such as Bcl-XL and decreases the expression of decoy receptors or pro-apoptotic genes such as DcR2 [11]. Hypoxia is an important pathway in CSCs and especially in niche, if hypoxia pathway is inhibited, the foundation of the life of cancer stem cells such as the survival and metastases and chemoresistance of cancer cells, will be insecure [12]. On the other hand, hypoxia pathway is under the control of Sphingosine kinase 1 or SPHK1 [13]. SPHK1 acts as a lipid kinase that phosphorylates sphingosine to sphingosine-1-phosphate (S1P) which is a new target in cancer therapy [14]. SPHK1 is capable of upregulating PI3K/mTOR/AKT pathway that causes cell survival and cell proliferation and it can also activates AKT and GSK3 $\beta$ then inactivates von Hippel-Lindau tumor suppressor protein (pVHL)-mediated ubiquitin proteasome machinery that a role in degredation of HIF-1 $\alpha$ (Fig. 1)[15].

High SPHK1 expression in CRC cell lines and tissue samples has been reported. Also, the key role of SPHK1 in tumor development and progression and its significant association with invasion and metastasis were confirmed [16]. So its role in CSC niche might be important. We hypothesize that if SPHK1 is knocked out in CSC niche as the microenvironment of CSCs, it could increase the response and the effect of chemotherapy with $5 \mathrm{fu}$ in CRC patients. Thus, SPHK1 knock out-based chemotherapies may represent a novel approach in CRC cell growth inhibition in early stages.

\section{Conclusion}

Chemotherapy resistant is a complex phenomenon that occurs in most cancers. No study to date has examined the effect of inhibition of SPHK1 with the targeting of hypoxia pathway in $\mathrm{CSC}$ niche in CRC to improve chemotherapy response. Thus, 


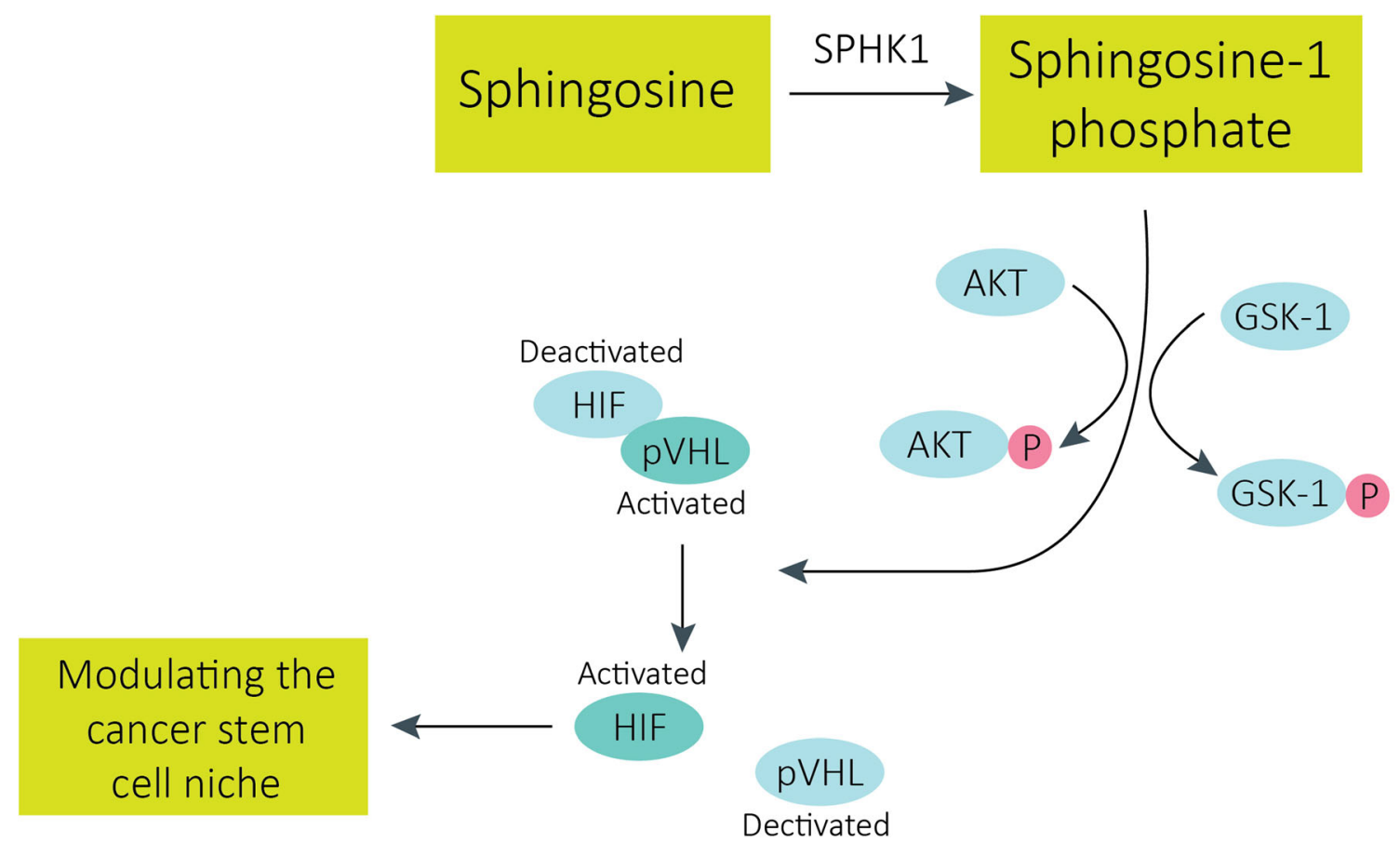

Fig. 1 SphK1 signaling pathway has been reported to modulate the expression of the important genes in cellular proliferation in CSC niche

if the results confirm our hypothesis, the SPHK1-mediated inhibition of HIF-1 to target the hypoxia pathway in CSC niche increases the effects of chemotherapy with 5-fu in CRC patients in early stages.

\section{Compliance with Ethical Standards}

Conflicts of Interest The authors have no conflicts of interest to declare.

\section{References}

1. Figer A, Perez-Staub N, Carola E, Tournigand C, Lledo G, Flesch $\mathrm{M}$, et al. FOLFOX in patients aged between 76 and 80 years with metastatic colorectal cancer. Cancer. 2007;110(12):2666-71.

2. Shim BY, Lee KM, Cho H-M, Kim HJ, Cho HJ, Yang J, et al. Oxaliplatin/5-FU without leucovorin chemotherapy in metastatic colorectal Cancer. Cancer Re Treat. 2005;37(4):212-5.

3. Samadi P, Afshar S, Amini R, Najafi R, Mahdavinezhad A, Sedighi Pashaki A, et al. Let-7e enhances the radiosensitivity of colorectal cancer cells by directly targeting insulin-like growth factor 1 receptor. 2018.

4. Chang L, Graham P, Hao J, Ni J, Deng J, Bucci J, et al. Cancer stem cells and signaling pathways in radioresistance. Oncotarget. 2016;7(10):11002.

5. Housman G, Byler S, Heerboth S, Lapinska K, Longacre M, Snyder N, et al. Drug resistance in cancer: an overview. Cancers. 2014;6(3):1769-92.

6. Frank NY, Schatton T, Frank MH. The therapeutic promise of the cancer stem cell concept. J Clin Invest. 2010;120(1):41-50.

7. Han L, Shi S, Gong T, Zhang Z, Sun X. Cancer stem cells: therapeutic implications and perspectives in cancer therapy. Acta Pharm Sin B. 2013;3(2):65-75.
8. Bao B, Azmi AS, Li Y, Ahmad A, Ali S, Banerjee S, et al. Targeting CSCs in tumor microenvironment: the potential role of ROSassociated miRNAs in tumor aggressiveness. Curr Stem Cell Res Ther. 2014;9(1):22-35.

9. O'Flaherty JD, Barr M, Fennell D, Richard D, Reynolds J, O’Leary $\mathrm{J}$, et al. The cancer stem-cell hypothesis: its emerging role in lung cancer biology and its relevance for future therapy. J Thorac Oncol. 2012;7(12):1880-90.

10. Malik S. Biotechnology and production of anti-cancer compounds: Springer; 2017.

11. Garza-Treviño EN, Said-Fernández SL, Martínez-Rodríguez HG. Understanding the colon cancer stem cells and perspectives on treatment. Cancer Cell Int. 2015;15(1):2.

12. De Francesco EM, Maggiolini M, Tanowitz HB, Sotgia F, Lisanti MP. Targeting hypoxic cancer stem cells (CSCs) with doxycycline: implications for optimizing anti-angiogenic therapy. Oncotarget. 2017;8(34):56126.

13. Takabe K, Paugh SW, Milstien S, Spiegel SJPr. "Inside-out" signaling of sphingosine-1-phosphate: therapeutic targets. 2008;60(2): 181-95.

14. Bouquerel P, Gstalder C, Müller D, Laurent J, Brizuela L, Sabbadini R, et al. Essential role for SphK1/S1P signaling to regulate hypoxia-inducible factor $2 \alpha$ expression and activity in cancer. 2016;5(3):e209.

15. Ader I, Malavaud B, Cuvillier O. When the sphingosine kinase 1/ sphingosine 1-phosphate pathway meets hypoxia signaling: new targets for cancer therapy. Cancer Res. 2009;69(9):3723-6.

16. Bae GE, Do S-I, Kim K, Park JH, Cho S, Kim H-SJAr. Increased Sphingosine Kinase 1 Expression Predicts Distant Metastasis and Poor Outcome in Patients With Colorectal Cancer. 2019;39(2):66370.

Publisher's Note Springer Nature remains neutral with regard to jurisdictional claims in published maps and institutional affiliations. 